\title{
Chaos Synchronization Based on Unknown Input Proportional Multiple-Integral Fuzzy Observer
}

\author{
T. Youssef, ${ }^{1,2}$ M. Chadli, ${ }^{1}$ H. R. Karimi, ${ }^{3}$ and M. Zelmat ${ }^{2}$ \\ ${ }^{1}$ Laboratory of Modeling, Information \& Systems (MIS), University of Picardie Jules Verne (UPJV), 33 rue Saint Leu, \\ 80039 Amiens Cedex 1, France \\ ${ }^{2}$ Laboratory of Automatic Applied (LAA), M'hamed Bougara University of Boumerdès (UMBB), 35000 Boumerdès, Algeria \\ ${ }^{3}$ Department of Engineering, Faculty of Engineering and Science, University of Agder, 4898 Grimstad, Norway
}

Correspondence should be addressed to M. Chadli; mchadli@u-picardie.fr and H. R. Karimi; hamid.r.karimi@uia.no

Received 1 April 2013; Revised 17 June 2013; Accepted 18 June 2013

Academic Editor: Zidong Wang

Copyright @ 2013 T. Youssef et al. This is an open access article distributed under the Creative Commons Attribution License, which permits unrestricted use, distribution, and reproduction in any medium, provided the original work is properly cited.

This paper presents an unknown input Proportional Multiple-Integral Observer (PIO) for synchronization of chaotic systems based on Takagi-Sugeno (TS) fuzzy chaotic models subject to unmeasurable decision variables and unknown input. In a secure communication configuration, this unknown input is regarded as a message encoded in the chaotic system and recovered by the proposed PIO. Both states and outputs of the fuzzy chaotic models are subject to polynomial unknown input with $k$ th derivative zero. Using Lyapunov stability theory, sufficient design conditions for synchronization are proposed. The PIO gains matrices are obtained by resolving linear matrix inequalities (LMIs) constraints. Simulation results show through two TS fuzzy chaotic models the validity of the proposed method.

\section{Introduction}

It is well known that the chaotic systems have a complex dynamical behavior and their fundamental characteristic is the chaos. The chaotic systems are highly sensitive to parameters variation and to initial conditions because the chaos is a source of oscillation and instability. Moreover, in the long term the behavior of the chaotic systems becomes difficult to predict which can lead systems to instability and undesirable performance [1]. On the other hand, the chaotic systems constitute a good platform to investigate the nonstandard control problems including synchronization and stabilization.

Since Pecora and Carroll [2, 3] have introduced in 1990 the concept of chaotic synchronization between two chaotic dynamical systems based on the Lorenz's chaotic system [4], the synchronization and control of chaotic systems attract more and more attention from various disciplines. A great deal of chaos applications have been developed in engineering fields such as secure communication, physical systems, system identification, and biological systems; see, for example, [5-10].

Recently, a particular attention has been paid to the synchronization and control problems for dynamical networks due to their extensive application in fields of science and engineering (see, e.g., [11-13]). In [11] the authors propose a novel concept of bounded $H_{\infty}$ synchronization and state estimation to handle the time-varying nature of an array stochastic complex network in discrete-time domain over a finite horizon. The synchronization problem in [12] is considered for a new class of continuous-time neural networks of neutral type with parameters, discrete-time delays, and unbounded distributed time delays being all dependent on the Markovian jumping mode. The sampled-data synchronization control scheme in [13] is studied for a class of dynamical networks with stochastic sampling. In the formwork of state estimation, fault detection, and filtering for a class of nonlinear systems with sensor networks, we can mention the works of [14-17]. Indeed, in [14] the finite-horizon 
distributed $H_{\infty}$ state estimator design scheme is proposed for a class of discrete time-varying nonlinear systems with both stochastic parameters and stochastic nonlinearities. The problem of designing the distributed $H_{\infty}$ filters in [15] is considered for a class of polynomial nonlinear stochastic systems which are represented in a state-dependent linearlike form. The distributed finite-horizon filter is proposed in [16] for a class of time-varying systems subject to randomly varying nonlinearities over lossy sensor networks that involve both the quantization errors and successive packet dropouts. The robust fuzzy fault detection filter in [17] is designed for a class of uncertain discrete-time Takagi-Sugeno fuzzy systems with successive packet dropouts which involve both the stochastic multiple time-varying discrete delays and the infinite distributed delays.

Different methods and techniques for chaos synchronization and control have been investigated including impulsive control [18], feedback control [19], adaptive control [20], lag synchronization [21], sliding mode control [22], and fuzzy control [23]. Particularly, in the chaotic secure communication problems, some messages can be masked efficiently and securely [24] by chaotic signals since the chaos has the characteristic of broadband like a noise and is consequently difficult to predict. The message in the secure communications which is recovered by the response system should synchronize with the drive system [25]. In framework of a secure communication, many approaches have been addressed such as chaos modulation [26], chaos shift key [27], and chaos masking [28]. Among different methods of synchronization and control for chaotic systems, the TS fuzzy systems have received much attention from various researches fields since the pioneering work of Takagi and Sugeno (TS) [29]. Indeed, TS fuzzy model can approximate a highly nonlinear analytical relation of chaotic system by fuzzy IF-THEN rules where the implications describe local dynamics as linear models. Then, the nonlinear behavior of the chaotic system can exactly be obtained as an aggregation of local linear models with nonlinear activation functions. TS fuzzy models are widely used as a tool of analysis and design of synchronization and control schemes because of the mathematical analysis simplicity of their simple structure with local dynamics, for example, [30-32].

Since because of, many practical control problems the states are partially or fully unavailable, the state observer methods can be used to estimate the measurements of unavailable or failed sensors. For this reason, it is important to design the observers for state estimation. In relation to that state estimation observers there are many works to that deal with stability analysis and stabilization of TS fuzzy models by applying Lyapunov theory and derive stability conditions in terms linear matrix inequalities constraints [33]; of among this works we can mention the results developed in [34]; When the decision variable is chosen as unmeasurable or unavailable state in activation functions, for example, in [35], the robust observer is designed for unknown inputs TS fuzzy models. Recently, in secure communication field the design problem of unknown input observer has been investigated in [36-39]. The authors propose in [36] a new secured transmission scheme based on smooth adaptive unknown input observers for chaotic synchronization and robust to channel noise. The unknown input observer in [37] is presented with unknown constant disturbance of parameters and unknown input to be recovered as messages in the master-slave configuration. The robust adaptive high-gain fuzzy observer is designed in [32] for chaotic systems where their parameters are assumed unknown and their states unavailable. The author deals with, in [38], the unknown input observer design for fuzzy systems with application to chaotic system reconstruction within both domains, continuous and discrete time, where the sufficient conditions have been derived in terms of linear matrix inequality constraints by using Lyapunov stability theory. Then, in $[38,39]$ the pole assignment in a LMI region is considered in order to improve the observer performance.

In the context of the unmeasurable decision variable, the synchronization problem for chaotic systems characterized by TS fuzzy models was not addressed by the above works. Our main contribution in this paper is to develop a synchronization procedure which takes into account the unmeasurable decision variables. The effects due to unmeasurable decision variable and the unknown input on the overall synchronization system (chaotic system and observer) are compensated with additional parameter. In addition, in the present study the estimation of the unknown input as a message to be reconstructed within a secure communication concept is considered.

In the framework of a secure communication, the design of unknown input PIO is addressed in this work for two chaotic systems, Lorenz' system and Rossler's system, characterized by chaotic TS fuzzy models. These models are subject to unmeasurable decision variable and polynomial unknown input where its $k$ th derivative is zero. The proposed PIO estimates both the states of the considered chaotic systems and the polynomial unknown input. This latter is considered as a message to encode by the chaotic system and then to reconstruct it by the PIO. Furthermore, the integral action included in the observer structure contributes to reduce the results conservatism due to quadratic function. Indeed, this parameter allows introducing an additional degree of freedom to be determined. By utilizing Lyapunov stability theory, sufficient conditions are derived to design the polynomial unknown input PIO. Then, the PIO gains parameters are resolved in terms of linear matrix inequalities constraints. Moreover, when the decision variables are measurable we also discuss this particular case in our work. Finally, we present simulation results to illustrate the effectiveness of the proposed approach of synchronization and reconstruction.

The rest of this paper is organized as follows. In Section 2, the considered unknown input TS fuzzy model structure is described. This unknown input affects both the dynamics of the TS fuzzy model and the output signal. The unknown input PIO is designed in Section 3. In Section 4, simulation results for two chaotic systems, Lorenz' system and Rossler's system, are given. An unknown input, assumed as a message to be received in a secure communication configuration, is perfectly reconstructed. Finally, a conclusion and further works end this paper. 


\section{Unknown Input TS Fuzzy Model Structure}

The TS fuzzy model subject to unknown input is considered as follow:

$$
\begin{gathered}
\dot{x}(t)=\sum_{i=1}^{r} \mu_{i}(x)\left(A_{i} x(t)+B_{i} u(t)+d_{i}+F_{i} v(t)\right), \\
y(t)=C x(t)+F v(t),
\end{gathered}
$$

where $x(t) \in R^{n}$ represents the state vector, $u(t) \in R^{n_{u}}$ corresponds to known input vector, $v(t) \in R^{n_{v}}$ shows the unknown input, and $y(t) \in R^{n_{y}}$ is the output vector. $A_{i} \in$ $R^{n \times n}$ are the state matrices, $B_{i} \in R^{n \times n_{u}}$ are the input matrices, $d_{i} \in R^{n}$ is a vector system dependent, $F_{i} \in R^{n \times n_{v}}$ and $F \in$ $R^{n_{y} \times n_{v}}$ are the unknown input matrices, and $C \in R^{n_{y} \times n}$ is the output matrix. The activation functions $\mu_{i}(x)$ depend on the state $x(t)$ of the system and satisfy the following conditions:

$$
\begin{gathered}
\sum_{i=1}^{r} \mu_{i}(x)=1, \quad \forall t \geq 0, \\
0 \leq \mu_{i}(x) \leq 1, \quad \forall i \in\{1, \ldots, r\},
\end{gathered}
$$

where $r$ is the number of local models.

Hypothesis 1. Unknown input $v(t)$ has a polynomial form of $k-1$ degree in time whose $k$ th derivative is equal to zero.

Let the following notations be introduced:

$$
\begin{gathered}
\dot{v}(t)=v_{1}(t), \\
\dot{v}_{1}(t)=v_{2}(t), \\
\vdots \\
\dot{v}_{k-1}(t)=v_{k}(t), \\
v_{k}(t)=0 .
\end{gathered}
$$

Note that the polynomial form allows considering a wide variety of unknown inputs.

\section{Unknown Input Proportional Multiple-Integral Observer Design}

The unknown input PIO is considered as follow:

$$
\begin{gathered}
\dot{\hat{x}}(t)=\sum_{i=1}^{r} \mu_{i}(\widehat{x})\left(A_{i} \widehat{x}(t)+B_{i} u(t)+d_{i}+F_{i} \widehat{v}(t)\right. \\
\left.+K_{P i}(y(t)-\widehat{y}(t))\right)+z_{x}(t), \\
\hat{y}(t)=C \widehat{x}(t)+F \widehat{v}(t), \\
\dot{\hat{v}}(t)=\sum_{i=1}^{r} \mu_{i}(\widehat{x}) K_{I i}(y(t)-\widehat{y}(t))+\widehat{v}_{1}(t)+z_{v}(t), \\
\dot{\hat{v}}_{j}(t)=\sum_{i=1}^{r} \mu_{i}(\widehat{x}) K_{I i}^{j}(y(t)-\widehat{y}(t))+\widehat{v}_{j+1}(t) \\
+z_{v j}(t), \quad \text { for } j: 1, \ldots, k-1 \\
\text { if } j=k-1, \quad \widehat{v}_{j+1}(t)=0,
\end{gathered}
$$

where $K_{P i} \in R^{n \times n_{y}}, K_{I i} \in R^{n_{v} \times n_{y}}$, and $K_{I i}^{j} \in R^{n_{v} \times n_{y}}$ correspond to proportional and integral gains, respectively. Due the effect of the unmeasurable decision variables, the variables $z_{x}(t)$, $z_{v}(t)$, and $z_{v j}(t)$ are introduced in the PIO.

According to Hypothesis 1, TS fuzzy model (1) and unknown input PIO (4) can be rewritten, respectively, as follow:

$$
\begin{gathered}
\dot{x}_{a}(t)=\sum_{i=1}^{r} \mu_{i}(x)\left(\bar{A}_{i} x_{a}(t)+\bar{B}_{i} u(t)+\bar{d}_{i}\right) \\
y(t)=\bar{C} x_{a}(t) \\
\dot{\hat{x}}_{a}(t)=\sum_{i=1}^{r} \mu_{i}(\widehat{x})\left(\bar{A}_{i} \widehat{x}_{a}(t)+\bar{B}_{i} u(t)+\bar{d}_{i}+\bar{K}_{i}(y(t)-\widehat{y}(t))\right) \\
+z(t), \quad \\
\hat{y}(t)=\bar{C} \widehat{x}_{a}(t),
\end{gathered}
$$

where

$$
\begin{gathered}
x_{a}(t)=\left[\begin{array}{c}
x(t) \\
v(t) \\
v_{1}(t) \\
\cdots \\
v_{k-1}(t)
\end{array}\right], \quad \widehat{x}_{a}(t)=\left[\begin{array}{c}
\widehat{x}(t) \\
\widehat{v}(t) \\
\widehat{v}_{1}(t) \\
\cdots \\
\widehat{v}_{k-1}(t)
\end{array}\right], \\
z(t)=\left[\begin{array}{c}
z_{x}(t) \\
z_{v}(t) \\
z_{v 1}(t) \\
\cdots \\
z_{v k-1}(t)
\end{array}\right]
\end{gathered}
$$

with

$$
\begin{gathered}
e_{a}(t)=x_{a}(t)-\hat{x}_{a}(t), \quad e_{a y}=y(t)-\hat{y}(t), \\
\bar{A}_{i}=\left[\begin{array}{cccccc}
A_{i} & F_{i} & 0 & 0 & \cdots & 0 \\
0 & 0 & I_{n_{v}} & 0 & \cdots & 0 \\
0 & 0 & 0 & I_{n_{v}} & \cdots & 0 \\
\cdots & \cdots & \cdots & \cdots & \cdots & I_{n_{v}} \\
0 & 0 & 0 & 0 & 0 & 0
\end{array}\right], \\
\bar{B}_{i}=\left[\begin{array}{c}
B_{i} \\
0 \\
0 \\
\cdots \\
0
\end{array}\right], \\
\bar{K}_{i}=\left[\begin{array}{c}
\bar{d}_{i}=\left[\begin{array}{c}
d_{i} \\
0 \\
0 \\
\cdots \\
K_{P i} \\
K_{I i}^{1} \\
K_{I i}^{1} \\
\cdots \\
K_{I i}^{k-1}
\end{array}\right], \\
\bar{C}
\end{array}\right],\left[\begin{array}{lllll}
C & F & 0 & \cdots
\end{array}\right],
\end{gathered}
$$

where $I_{n_{v}}$ is an identity matrix. 
3.1. Unmeasurable Decision Varaiables. The dynamic error $e_{a}(t)$ of state estimation is given by

$$
\dot{e}_{a}(t)=\sum_{i=1}^{r} \mu_{i}(\widehat{x}) \overline{\mathscr{A}}_{i} e_{a}(t)+\bar{\Delta} A x_{a}(t)+\bar{\Delta} B u(t)+\bar{\Delta} d-z(t),
$$

where

$$
\begin{gathered}
\overline{\mathscr{A}}_{i}=\bar{A}_{i}-\bar{K}_{i} \bar{C}, \quad \bar{\Delta} A=\sum_{i=1}^{r} \bar{\mu}_{i} \bar{A}_{i}, \quad \overline{\Delta B}=\sum_{i=1}^{r} \bar{\mu}_{i} \bar{B}_{i}, \\
\bar{\Delta} d=\sum_{i=1}^{r} \bar{\mu}_{i} \bar{d}_{i}, \quad \overline{\mu_{i}}=\mu_{i}(x)-\mu_{i}(\widehat{x}) .
\end{gathered}
$$

Remark 1 . Since the activation functions satisfy the convex sum property, we can write $-1<\overline{\mu_{i}}<1$, and the variables matrices $\bar{\Delta} A, \bar{\Delta} B, \bar{\Delta} d$ are bounded and the following conditions hold:

$$
\begin{array}{cc}
\|\bar{\Delta} A\| \leq \delta_{1}, & \delta_{1}=\sum_{i=1}^{r} \delta_{1 i}, \quad\|\bar{\Delta} B\| \leq \delta_{2}, \\
\delta_{2}=\sum_{i=1}^{r} \delta_{2 i}, & \|\bar{\Delta} d\| \leq \delta_{3}, \quad \delta_{3}=\sum_{i=1}^{r} \delta_{3 i}
\end{array}
$$

with $\delta_{1 i}>0, \delta_{2 i}>0$ and $\delta_{3 i}>0$, are the Euclidian norms of $\bar{A}_{i}, \bar{B}_{i}$, and $\bar{d}_{i}$, respectively.

Theorem 2. The dynamic error (8) is asymptotically stable if there exist a common positive definite matrix $P=P^{T}$, matrices $\bar{M}_{i}$ and the positive scalars $\alpha$ and $\alpha_{0}$, for all $i \in\{1, \ldots, r\}$, such that:

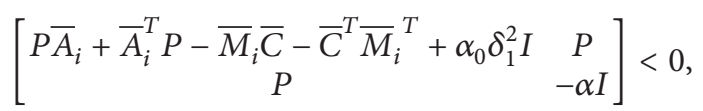

where the matrices and parameters $\bar{A}_{i}, \bar{C}, \delta_{1}$ are defined in $(7 \mathrm{c})$, and (10), respectively.

The parameters of unknown input PIO (4) are obtained by

$$
\begin{gathered}
\bar{K}_{i}=P^{-1} \bar{M}_{i}, \\
z=0, \quad \text { if }\left|e_{a y}\right|<\varepsilon, \\
z=\sigma_{1} \delta_{1}^{2} \frac{\widehat{x}_{a}^{T} \widehat{x}_{a}}{2 e_{a y}^{T} e_{a y}} P^{-1} \bar{C}^{T} e_{a y}+\sigma_{2} \delta_{2}^{2} \frac{u^{T} u}{2 e_{a y}^{T} e_{a y}} P^{-1} \bar{C}^{T} e_{a y} \\
+\sigma_{3} \delta_{3}^{2} \frac{1}{2 e_{a y}^{T} e_{a y}} P^{-1} \bar{C}^{T} e_{a y}, \quad \text { if }\left|e_{a y}\right| \geq \varepsilon
\end{gathered}
$$

with variables $\hat{x}_{a}, z, e_{a y}$ and the parameters $\delta_{2}, \delta_{3}$ are described in (7a), (7b), (10), respectively, and $\sigma_{1}=\left(\alpha_{0} / \lambda\right)$, $\sigma_{2}=\left(\left(\alpha \alpha_{0} \lambda_{3}\right) /\left(\alpha\left(\alpha_{0}+\lambda_{3}(1+\lambda)\right)-\alpha_{0} \lambda_{3}\right)\right), \sigma_{3}=\lambda_{3}$ where $\lambda, \lambda_{3}$ are positive scalars arbitrarily fixed and $\varepsilon$ is a very small positive threshold.
Proof. The proposed quadratic function of Lyapunov is $V(t)=e_{a}^{T}(t) P e_{a}(t)$ with $P=P^{T}>0$. The conditions ((11a), (11b), and (11c)) guarantee the asymptotic stability of the dynamic error of state estimation (8). The proof is partially given in the appendix and for more details see [40].

3.2. Measurable Decision Variables Case. The design of unknown input PIO with measurable decision variables represents the particular case of our study developed in this section. In this condition the unknown input PIO is as follow:

$$
\begin{gathered}
\dot{\hat{x}}(t)=\sum_{i=1}^{r} \mu_{i}(x)\left(A_{i} \widehat{x}(t)+B_{i} u(t)+d_{i}+F_{i} \widehat{v}(t)\right. \\
\left.+K_{P i}(y(t)-\widehat{y}(t))\right), \\
\widehat{y}(t)=C \widehat{x}(t)+F \widehat{v}(t), \\
\dot{\hat{v}}(t)=\sum_{i=1}^{r} \mu_{i}(x) K_{I i}(y(t)-\widehat{y}(t))+\widehat{v}_{1}(t), \\
\dot{\hat{v}}_{j}(t)=\sum_{i=1}^{r} \mu_{i}(x) K_{I i}^{j}(y(t)-\widehat{y}(t))+\widehat{v}_{j+1}(t), \\
\quad \text { for } j: 1, \ldots, k-1 \\
\text { if } j=k-1, \quad \widehat{v}_{j+1}(t)=0,
\end{gathered}
$$

where all variables and matrices are defined in relations $((7 \mathrm{a})$, (7b), and (7c)), and the activation functions $\mu_{i}(x)$ depend on the measurable states.

The dynamics of the augmented state estimation error $e_{a}(t)$ between the TS fuzzy model (1) and PIO (12) becomes

$$
\dot{e}_{a}(t)=\sum_{i=1}^{r} \mu_{i}(x)\left(\bar{A}_{i}-\bar{K}_{i} \bar{C}\right) e_{a}(t) .
$$

Theorem 3. The dynamic error (13) is asymptotically stable if there exist a symmetric matrix $Q>0$ and matrices $\bar{N}_{i}$ such that the following conditions hold, for all $i \in\{1, \ldots, r\}$ :

$$
Q \bar{A}_{i}+\bar{A}_{i}^{T} Q-\bar{N}_{i} \bar{C}-\bar{C}^{T} \bar{N}_{i}^{T}<0 .
$$

The parameters of the unknown input PIO (12) are given by:

$$
\bar{K}_{i}=Q^{-1} \bar{N}_{i}
$$

Proof. Consider the Lyapunov quadratic function $V(t)=$ $e_{a}^{T}(t) Q e_{a}(t)$, where $Q=Q^{T}>0$. The time derivative of $V(t)$ allows writing

$$
\dot{V}=\sum_{i=1}^{r} \mu_{i}(\widehat{x}) e_{a}^{T}\left(\left(\bar{A}_{i}-\bar{K}_{i} \bar{C}\right)^{T} Q+Q\left(\bar{A}_{i}-\bar{K}_{i} \bar{C}\right)_{i}\right) e_{a} .
$$

The stability condition $\dot{V}(t)<0$ is satisfied if

$$
\left(\bar{A}_{i}-\bar{K}_{i} \bar{C}\right)^{T} Q+Q\left(\bar{A}_{i}-\bar{K}_{i} \bar{C}\right)_{i}<0 ;
$$

with variables change $\bar{N}_{i}=Q \bar{K}_{i}$; we obtain the linear matrix inequalities ((14a) and (14b)). The proof is completed. 


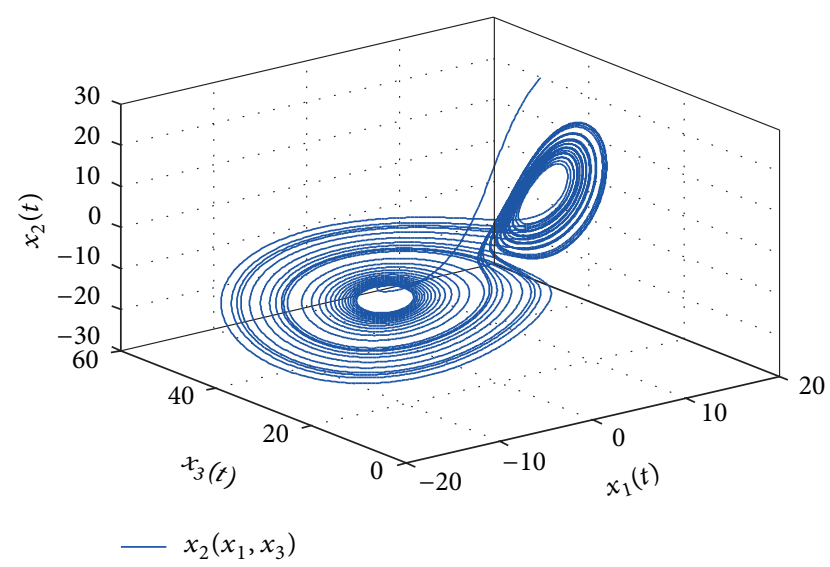

FIGURE 1: Chaotic behavior of Lorenz fuzzy system.

The unknown input PIO gains $\bar{K}_{i}=Q^{-1} \bar{N}_{i}$ are determined by resolving these constraints. In the following section, a simulation example is given through two chaotic systems in order to validate this proposed approach.

\section{Simulation Examples}

Two chaotic systems are characterized by TS fuzzy models with unmeasurable decision variables and subjected to unknown input. In a secure communication concept, the unknown input is assumed as a message to be recovered in the PIO after being encoded in the chaotic system by means of public transmission canal. These chaotic systems are used to show the good simultaneous reconstruction of states and message by the proposed unknown input PIO. The first nonlinear model is the Lorenz's system [41], and the second is the fourth Rossler's system [21].

4.1. Lorenz Chaotic System. The Lorenz chaotic system [38] is represented by following the dynamic equations:

$$
\begin{gathered}
\dot{x}_{1}=-10 x_{1}+10 x_{2}, \\
\dot{x}_{2}=28 x_{1}-x_{2}-x_{1} x_{3}, \\
\dot{x}_{3}=x_{1} x_{2}-\frac{8}{3} x_{3} .
\end{gathered}
$$

4.1.1. TS Fuzzy Model. The Lorenz's system can be exactly represented by TS fuzzy model with the decision variable $x_{1}(t) \in[-30,30]$ as follow [42]:

$$
\begin{aligned}
& \text { Rule 1: } x_{1}(t) \text { is } \mu_{1}\left(x_{1}(t)\right) \text {, THEN } \dot{x}(t)=A_{1} x(t), \\
& \text { Rule 2: } x_{2}(t) \text { is } \mu_{2}\left(x_{1}(t)\right) \text {, THEN } \dot{x}(t)=A_{2} x(t),
\end{aligned}
$$

where $x(t)=\left[x_{1}(t), x_{2}(t), x_{3}(t)\right], \mu_{1}\left(x_{1}(t)\right)=\left(30+x_{1}(t)\right) / 60$, $\mu_{2}\left(x_{1}(t)\right)=\left(30-x_{1}(t)\right) / 60$, and

$$
A_{1}=\left[\begin{array}{ccc}
-10 & 10 & 0 \\
28 & -1 & -30 \\
0 & 30 & \frac{-8}{3}
\end{array}\right], \quad A_{2}=\left[\begin{array}{ccc}
-10 & 10 & 0 \\
28 & -1 & 30 \\
0 & -30 & \frac{-8}{3}
\end{array}\right]
$$

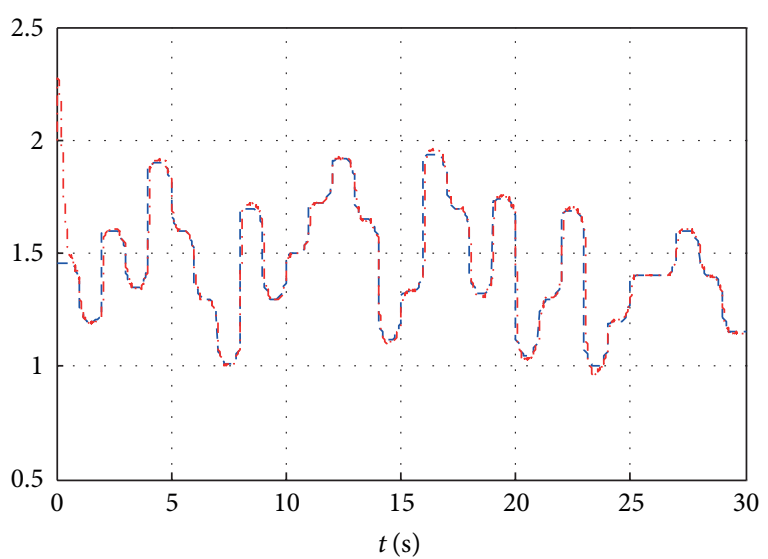

$---v$

Figure 2: The unknown input and its estimated.

TABLE 1

\begin{tabular}{lcc}
\hline$\lambda=2 \times 10^{3}$ & $\alpha=5.505 \times 10^{4}$ & $\alpha_{0}=0.001$ \\
$i$ & 1 & 2 \\
\hline$K_{p i}$ & {$\left[\begin{array}{ll}-11.471 & 12.445 \\
-22.084 & 10.838 \\
-94.350 & 96.029\end{array}\right]$} & {$\left[\begin{array}{cc}-11.057 & 11.926 \\
02.100 & 11.650 \\
97.909 & -96.239\end{array}\right]$} \\
$K_{I i}$ & {$\left[\begin{array}{ll}15.123 & 03.344\end{array}\right]$} & {$\left[\begin{array}{ll}21.570 & -03.137\end{array}\right]$} \\
$K_{I i}^{1}$ & {$\left[\begin{array}{ll}83.889 & -02.802\end{array}\right]$} & {$\left[\begin{array}{ll}79.394 & 01.612\end{array}\right]$} \\
\hline
\end{tabular}

The Lorenz chaotic attractor is given in Figure 1.

The TS fuzzy model of the Lorenz chaotic system (17) is

$$
\begin{gathered}
\dot{x}(t)=\sum_{i=1}^{2} \mu_{i}(x)\left(A_{i} x(t)+E_{i} v(t)\right), \\
y(t)=C x(t)+E v(t),
\end{gathered}
$$

with $B_{1}=B_{2}=\left[\begin{array}{l}0 \\ 0 \\ 0\end{array}\right], E_{1}=\left[\begin{array}{l}1 \\ 1 \\ 1\end{array}\right], E_{2}=\left[\begin{array}{c}1 \\ 0.5 \\ 1\end{array}\right], E=\left[\begin{array}{l}1 \\ 1\end{array}\right], C=$ $\left[\begin{array}{lll}0 & 0 & 1 \\ 0 & 1 & 1\end{array}\right]$.

The unknown input $v(t)$ is assumed as a message to be recovered by the unknown input PIO (4). This observer plays the role of decoder and the chaotic system the encoder within a secure communication configuration.

4.1.2. Unknown Input PIO. The unknown input PIO gains are determined by resolving the LMIs constraints ((11a), (11b), and (11c)) of Theorem 2:

$$
\bar{K}_{i}=\left[\begin{array}{lll}
K_{P i}^{T} & K_{I i}^{T} & K_{I i}^{1 T}
\end{array}\right]^{T}, \quad Z=\left[\begin{array}{lll}
Z_{x}^{T} & Z_{v}^{T} & Z_{v 1}^{T}
\end{array}\right]^{T} .
$$

The unknown input PIO gains are given by Table 1 .

The unknown input is assumed as a message to be encoded by chaotic system with the second derivative being zero, shown in Figure 2. The simulation results are obtained 


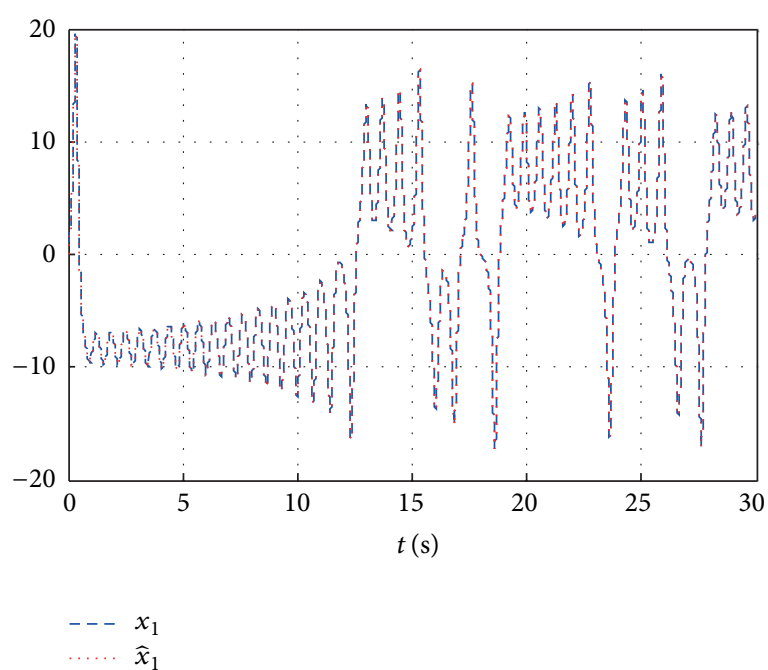

(a)

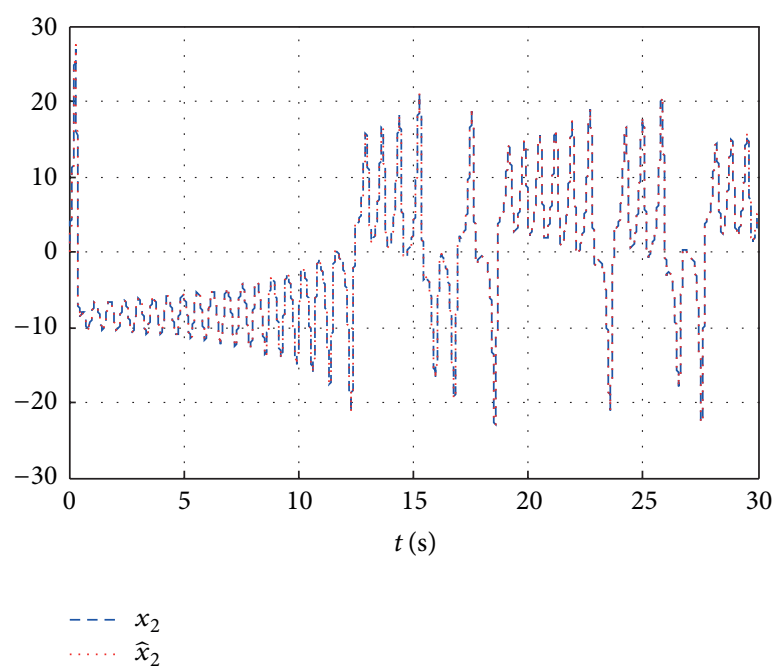

(b)

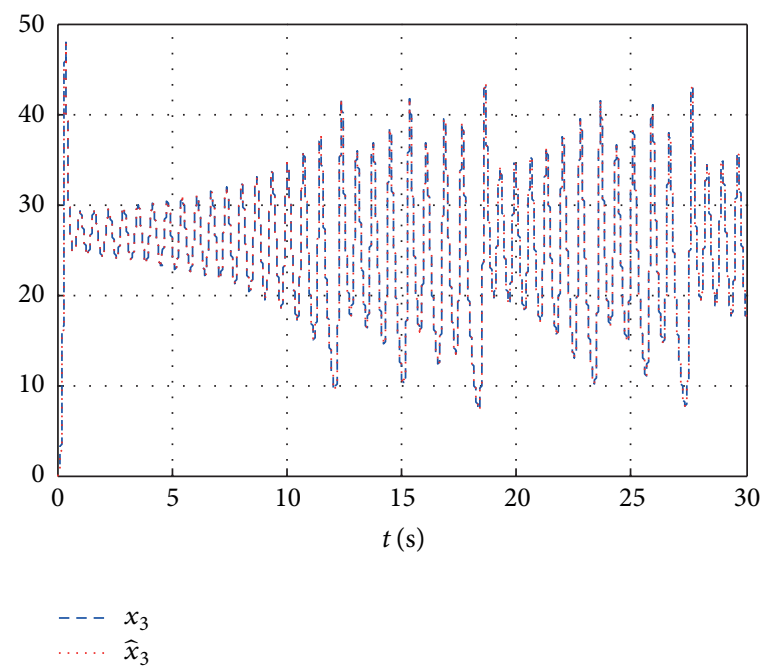

(c)

FIGURE 3: (a) The state $x_{1}(t)$ and its estimated $\hat{x}_{1}(t)$. (b) The state $x_{2}(t)$ and its estimated $\hat{x}_{2}(t)$. (c) The state $x_{3}(t)$ and its estimated $\widehat{x}_{3}(t)$.

with the initial conditions $x_{0}=\left[\begin{array}{lll}1 & 1 & 1\end{array}\right] \widehat{x}_{0}=\left[\begin{array}{lll}0 & 0 & 0\end{array}\right]$ and with $\varepsilon=10^{-3}$.

The unknown input and the estimated one are given in Figure 2. Excepted around the time origin, we obtained a good reconstruction of the unknown input. Figures 3(a), 3(b), and $3(\mathrm{c})$ show the states $x_{1}(t), x_{2}(t)$, and $x_{3}(t)$ and their estimated $\widehat{x}_{1}(t), \widehat{x}_{2}(t)$, and $\hat{x}_{3}(t)$, respectively.

The dynamic errors of the states estimation are given in Figure 4. The obtained simulation results show the good reconstruction of the states and the unknown input.

Remark 4. Note that applying Theorem 3 instead of Theorem 2 for this example leads to bad estimation. The simulation results for this example, carried out with the same initial conditions: $x_{0}=\left[\begin{array}{lll}1 & 1 & 1\end{array}\right]$ and $\widehat{x}_{0}=\left[\begin{array}{lll}0 & 0 & 0\end{array}\right]$, are shown in Figure 5. Indeed, we see clearly that the best estimation (Figure 6) is given by Theorem 2 (dashed line) which takes into account the estimation of decision variables.
4.2. Fourth Rossler Chaotic System. The fourth Rossler chaotic system [21] is represented by the following dynamic equations:

$$
\begin{gathered}
\dot{x}_{1}=-x_{2}-x_{3}, \\
\dot{x}_{2}=x_{1}+0,254 x_{2}+x_{4}, \\
\dot{x}_{3}=x_{1} x_{3}+3, \\
\dot{x}_{4}=-0,5 x_{3}+0,05 x_{4} .
\end{gathered}
$$

4.2.1. TS Fuzzy Model. The fourth Rossler's system can be exactly described by TS fuzzy model with the decision variable $x_{1}(t) \in[-80,20]$ as follow [42]:

Rule 1: $x_{1}(t)$ is $\mu_{1}\left(x_{1}(t)\right)$, THEN $\dot{x}(t)=A_{1} x(t)+d_{1}$, Rule 2: $x_{2}(t)$ is $\mu_{2}\left(x_{1}(t)\right)$, THEN $\dot{x}(t)=A_{2} x(t)+d_{2}$, 


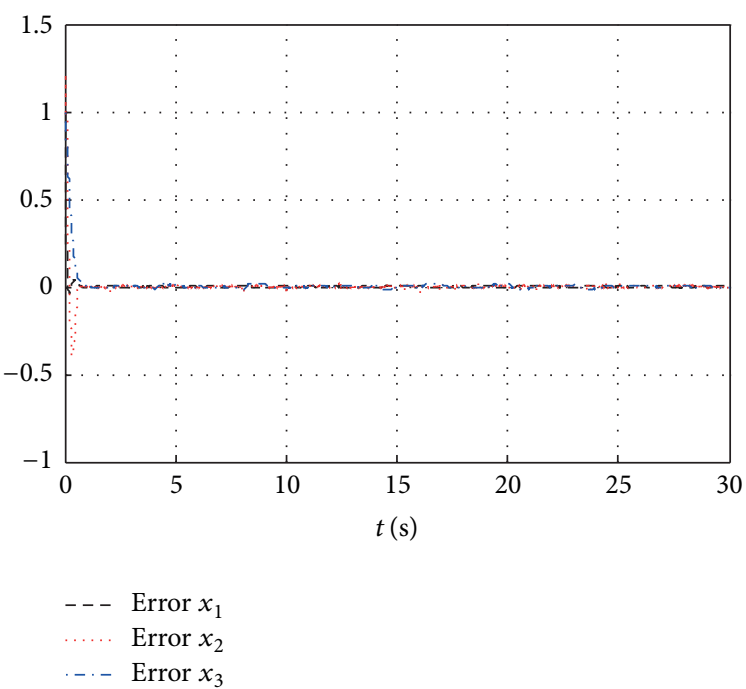

FIgURE 4: The errors between states and their estimated.

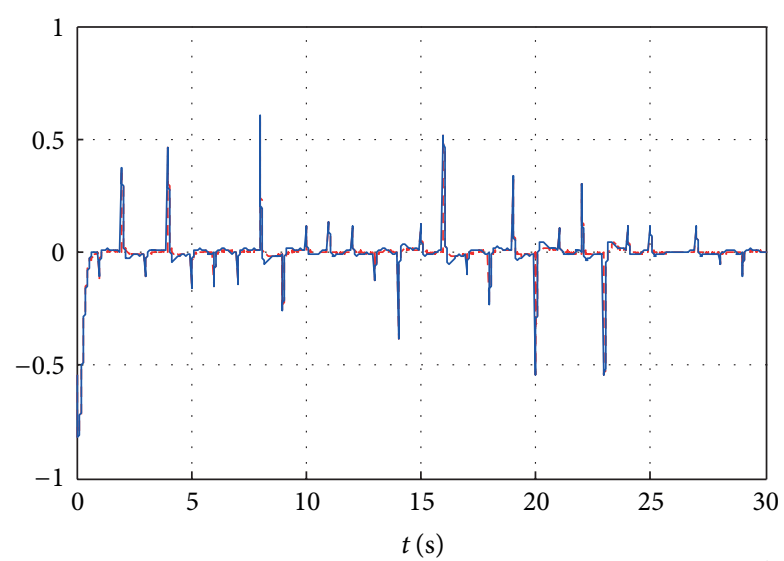

Error $v$ with Theorem 1

— Error $v$ with Theorem 2

FIGURE 5: The errors between unknown input and its estimated.

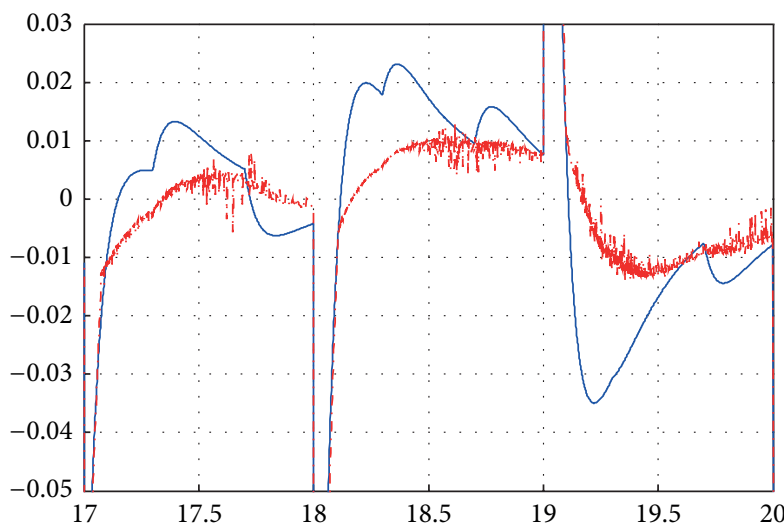

FIGURE 6: The zoom on the errors between unknown input and its estimated.
TABLE 2

\begin{tabular}{|c|c|c|c|c|}
\hline$\lambda=3 \times 10^{3}$ & \multicolumn{2}{|c|}{$\alpha=6.243 \times 10^{5}$} & \multicolumn{2}{|c|}{$\alpha_{0}=6.467 \times 10^{-5}$} \\
\hline$i$ & 1 & 1 & 2 & 2 \\
\hline \multirow{4}{*}{$K_{p i}$} & 011.018 & 001.805 & 020.387 & -026.055 \\
\hline & -000.343 & 001.344 & 004.339 & -013.805 \\
\hline & -078.779 & 109.443 & 166.122 & -242.987 \\
\hline & 011.208 & 005.138 & 028.076 & -045.031 \\
\hline$K_{I i}$ & 052.544 & -006.911 & 030.739 & 058.089 \\
\hline$K_{I i}^{1}$ & 097.452 & -008.136 & 066.736 & 083.503 \\
\hline$K_{I i}^{2}$ & 091.450 & -008.807 & 058.600 & 089.165 \\
\hline$K_{I i}^{3}$ & 034.284 & -001.174 & 027.830 & 018.118 \\
\hline
\end{tabular}

where $x(t)=\left[x_{1}(t), x_{2}(t), x_{3}(t), x_{4}(t)\right], \mu_{1}\left(x_{1}(t)\right)=(80+$ $\left.x_{1}(t)\right) / 100, \mu_{2}\left(x_{1}(t)\right)=\left(20-x_{1}(t)\right) / 100$, and

$$
\begin{aligned}
A_{1}= & {\left[\begin{array}{cccc}
0 & -1 & -1 & 0 \\
1 & 0.25 & 0 & 1 \\
0 & 0 & 20 & 0 \\
0 & 0 & -0.5 & 0.05
\end{array}\right], } \\
A_{2}= & {\left[\begin{array}{cccc}
0 & -1 & -1 & 0 \\
1 & 0.25 & 0 & 1 \\
0 & 0 & -80 & 0 \\
0 & 0 & -0.5 & 0.05
\end{array}\right], } \\
& d_{1}=d_{2}=\left[\begin{array}{l}
0 \\
0 \\
3 \\
0
\end{array}\right] .
\end{aligned}
$$

The fourth Rossler chaotic attractor is given in Figure 7.

The TS fuzzy model of the fourth Rossler system (21) is

$$
\begin{gathered}
\dot{x}(t)=\sum_{i=1}^{2} \mu_{i}(x)\left(A_{i} x(t)+E_{i} v(t)+d_{i}\right), \\
y(t)=C x(t)+E v(t)
\end{gathered}
$$

with $B_{1}=B_{2}=\left[\begin{array}{l}0 \\ 0 \\ 0 \\ 0\end{array}\right], E_{1}=\left[\begin{array}{l}1 \\ 1 \\ 1 \\ 1\end{array}\right], E_{2}=\left[\begin{array}{c}1 \\ 0.5 \\ 1 \\ 1\end{array}\right], E=\left[\begin{array}{l}1 \\ 1\end{array}\right]$, $C=\left[\begin{array}{llll}0 & 1 & 0 & 1 \\ 0 & 0 & 1 & 0\end{array}\right]$.

The unknown input $v(t)$ is assumed as a message to be encoded by the TS fuzzy model (23).

4.2.2. Unknown Input PIO. The unknown PIO gains are obtained by resolving the LMIs constraints ((11a), (11b), and (11c)) of Theorem 3:

$$
\begin{aligned}
\bar{K}_{i} & =\left[\begin{array}{lllll}
K_{P i}^{T} & K_{I i}^{T} & K_{I i}^{1 T} & K_{I i}^{2 T} & K_{I i}^{3 T}
\end{array}\right]^{T}, \\
Z & =\left[\begin{array}{lllll}
Z_{x}^{T} & Z_{v}^{T} & Z_{v 1}^{T} & Z_{v 2}^{T} & Z_{v 3}^{T}
\end{array}\right]^{T} .
\end{aligned}
$$

The unknown PIO gains are given by Table 2 .

The unknown input is assumed as a message to be encoded by chaotic system. The best results are obtained 


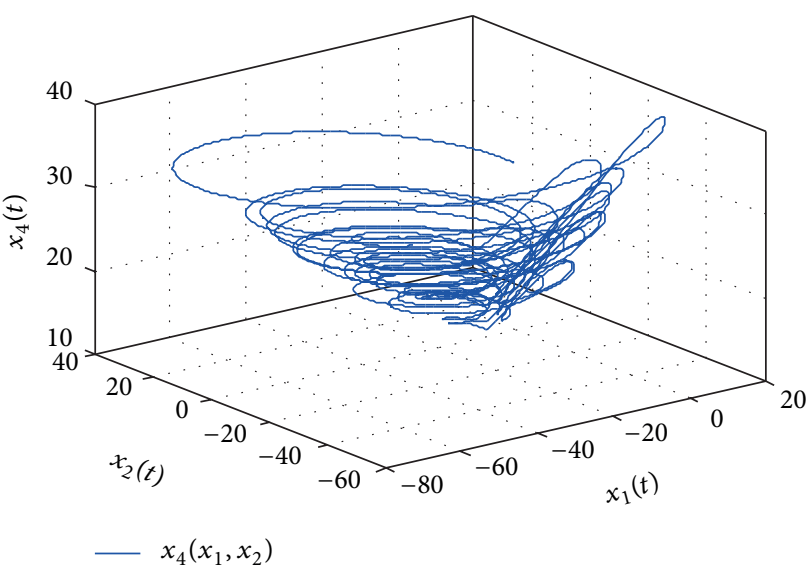

(a)

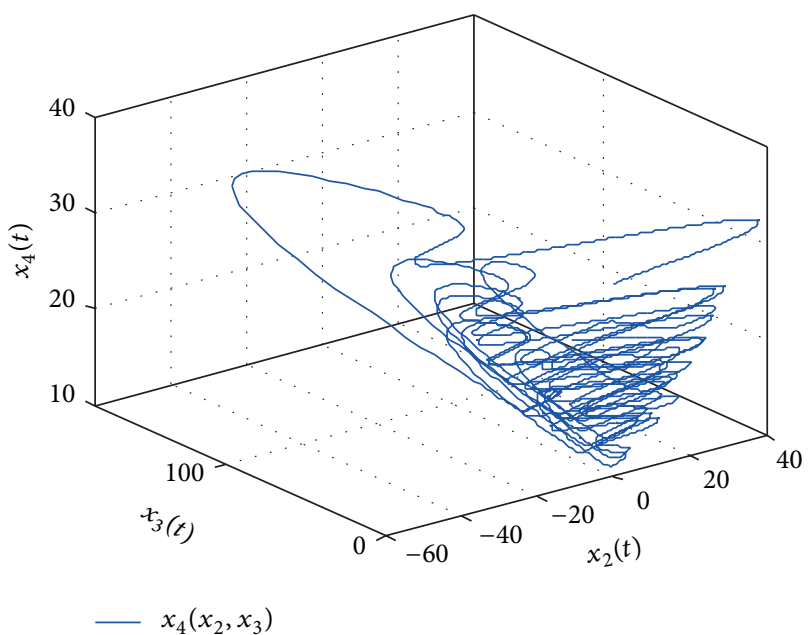

(c)

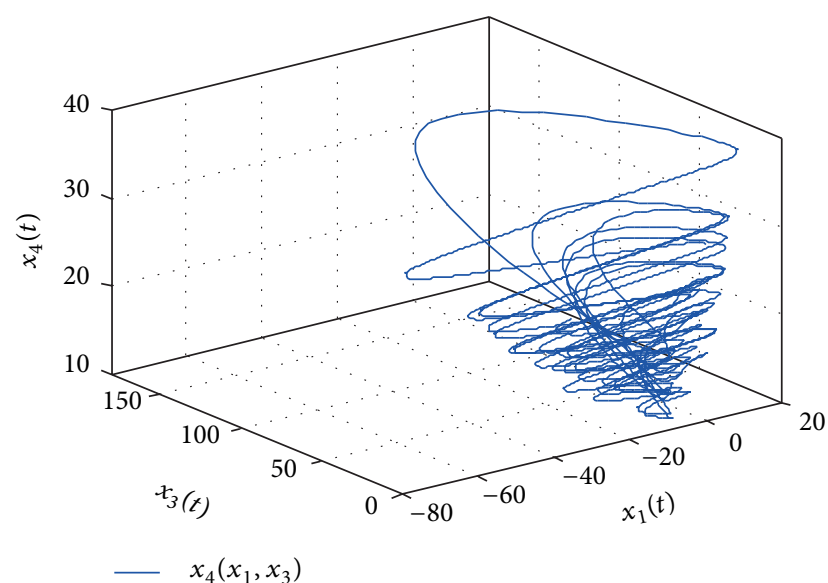

(b)

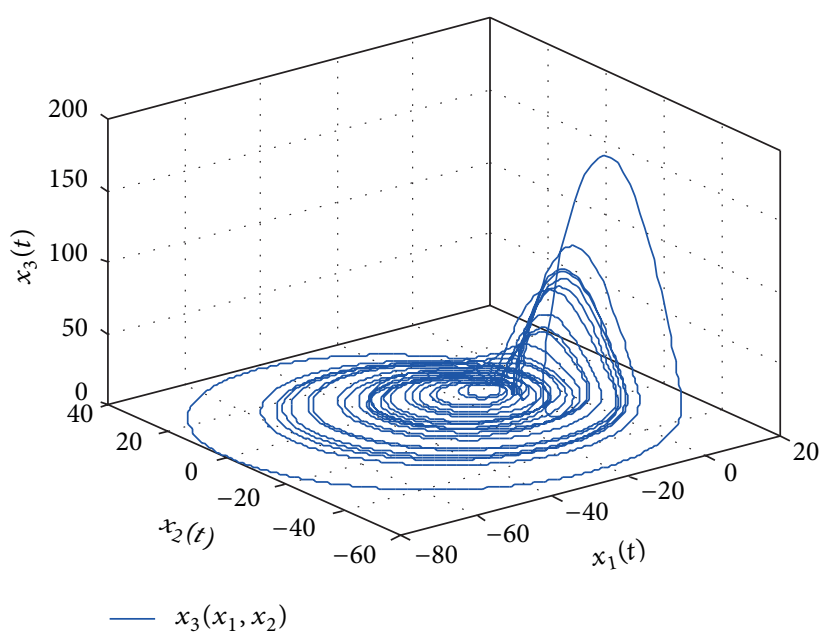

(d)

Figure 7: (a) Chaotic behavior $x_{4}\left(x_{1}(t), x_{2}(t)\right)$ of fourth Rossler fuzzy system. (b) Chaotic behavior $x_{4}\left(x_{1}(t), x_{3}(t)\right)$ of fourth Rossler fuzzy system. (c) Chaotic behavior $x_{4}\left(x_{2}(t), x_{3}(t)\right)$ of fourth Rossler fuzzy system. (d) Chaotic behavior $x_{3}\left(x_{1}(t), x_{2}(t)\right)$ of fourth Rossler fuzzy system.

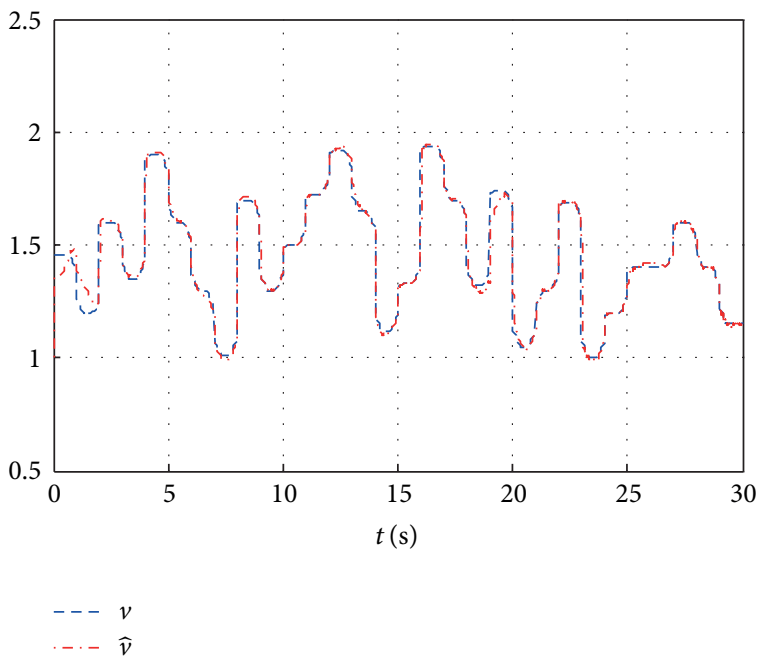

FIGURE 8: The unknown input and its estimated. with the fourth derivative being zero, shown in Figure 8. The simulation results are obtained with the initial conditions $x_{0}=\left[\begin{array}{llll}0 & 0 & 0 & 30\end{array}\right]$ and $\widehat{x}_{0}=\left[\begin{array}{llll}1 & 1 & 1 & 29\end{array}\right]$ and with $\varepsilon=10^{-3}$.

The unknown input and the estimated one are given in Figure 8. Excepted around the time origin, we got a good reconstruction of the unknown input. Figures 9(a), 9(b), 9(c) and $9(\mathrm{~d})$ represent the states $x_{1}(t), x_{2}(t), x_{3}(t)$, and $x_{4}(t)$ and their estimated $\widehat{x}_{1}(t), \widehat{x}_{2}(t), \widehat{x}_{3}(t)$, and $\widehat{x}_{4}(t)$, respectively.

The dynamic errors of the states estimation are represented in Figure 10. The obtained simulation results show the good estimation of the states and the unknown input.

\section{Conclusion}

In this paper, we have addressed the synchronization and reconstruction problem for chaotic systems. The TS fuzzy models subjected to unmeasurable decision variables and 


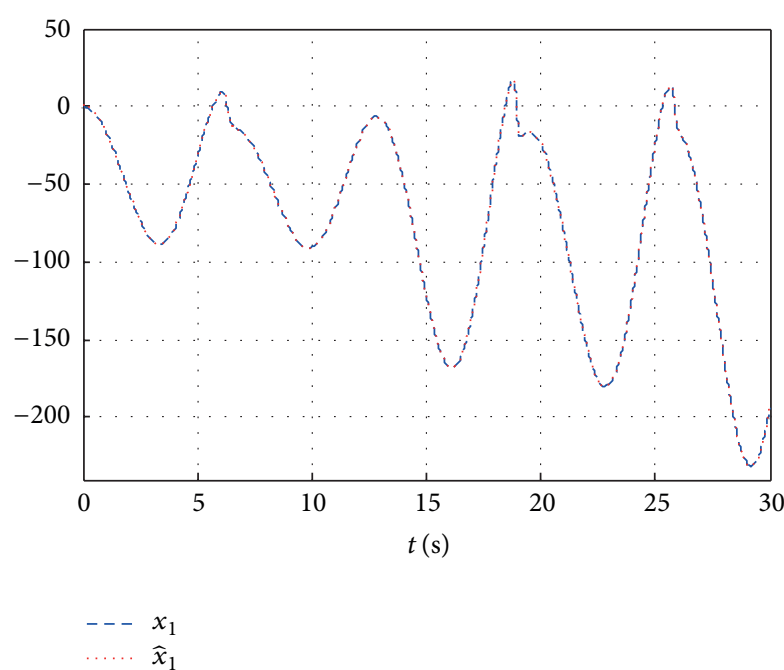

(a)

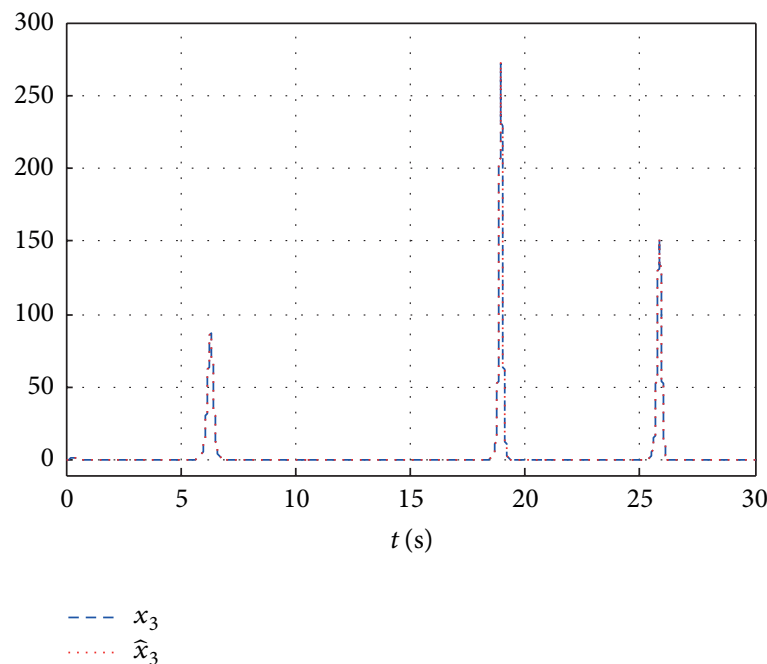

(c)

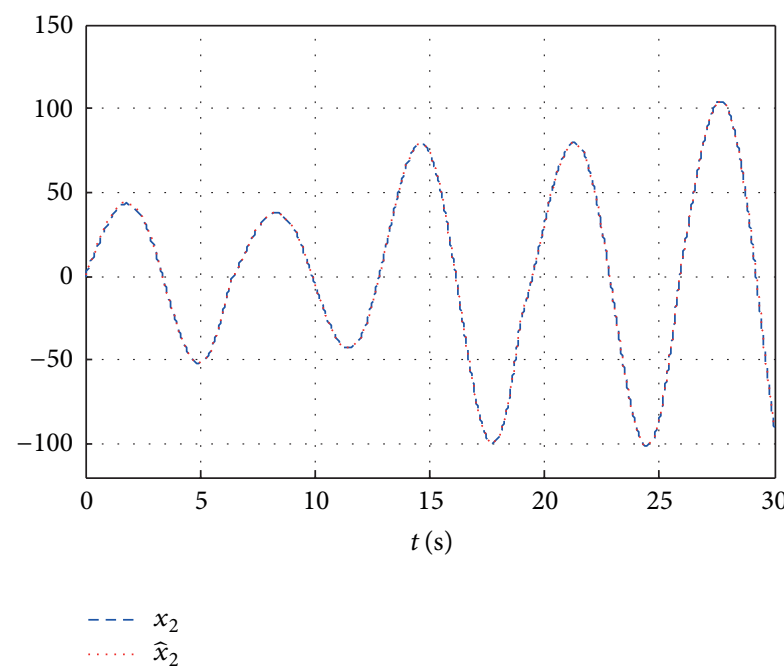

(b)

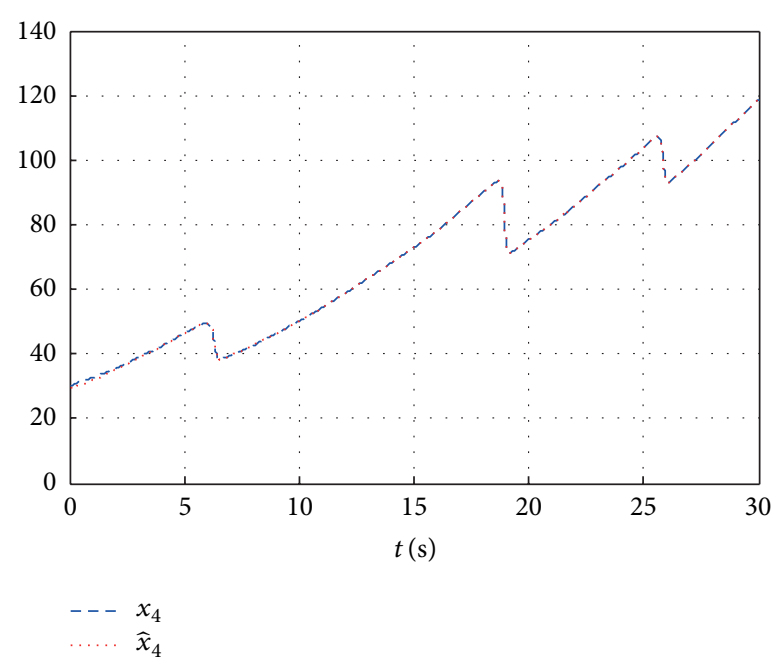

(d)

FIgure 9: (a) The state $x_{1}(t)$ and its estimated $\hat{x}_{1}(t)$. (b) The state $x_{2}(t)$ and its estimated $\hat{x}_{2}(t)$. (c) The state $x_{3}(t)$ and its estimated $\widehat{x}_{3}(t)$. (d) The state $x_{4}(t)$ and its estimated $\hat{x}_{4}(t)$.

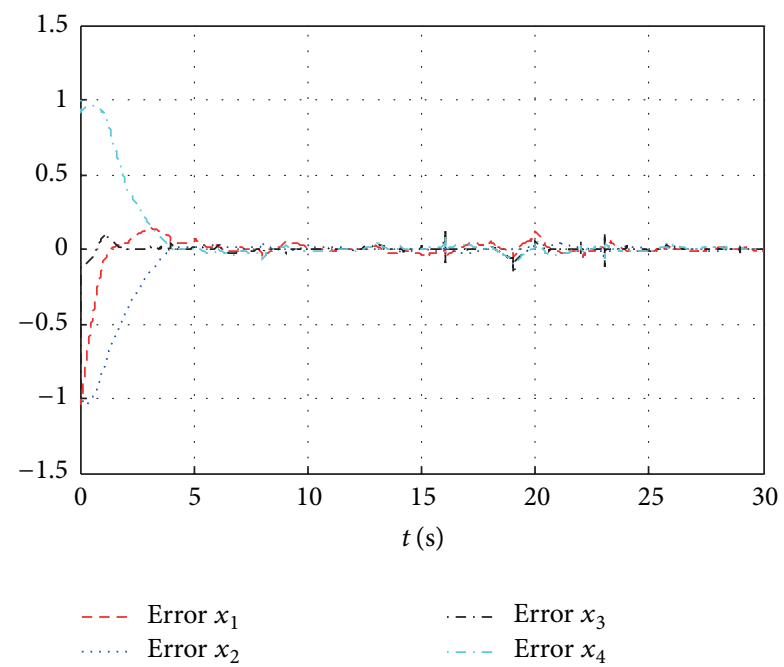

FIGURE 10: The errors between states and their estimated. unknown inputs are employed to exactly describe the behavior of two chaotic systems, Lorenz's system and Rossler's system. Based on Lyapunov theory and LMI formulation, an unknown input proportional integral observer to achieve the synchronization and the unknown input reconstruction is designed. To take into account a wide variety of unknown inputs, a polynomial form with $k$ th derivative zero is considered. Moreover, both the measurable and unmeasurable decision variables cases are studied. Simulation results are given to verify the effectiveness of the proposed method by reconstructing both states and unknown inputs. In the secure communication field, the proposed polynomial unknown input PIO with unmeasurable decision variables presents a good synchronization technique and messages recovering.

Motivated by the given results, the problem of diagnosis and fault tolerant control for more complex systems will be considered. Moreover, to reduce the conservatism due to the 
quadratic approach, nonquadratic Lyapunov functions will be introduced in our further research.

\section{Appendix}

By using the quadratic Lyapunov function $V(t)=e_{a}^{T}(t) P e_{a}(t)$ where $P=P^{T}>0$ and the following lemma.

Lemma A.1. For any matrices $X$ and $Y$ of appropriate dimensions, the following property is satisfied:

$$
X^{T} Y+Y^{T} X \leq \lambda X^{T} X+\lambda^{-1} Y^{T} Y \quad \text { with } \lambda>0,
$$

the time-derivative of $V(t)$ leads

$$
\begin{gathered}
\dot{V} \leq \sum_{i=1}^{r} \mu_{i}(\widehat{x})\left(e_{a}^{T}\left(\overline{\mathscr{A}}_{i}^{T} P+P \overline{\mathscr{A}}_{i}+\alpha_{0} \delta_{1}^{2} I+\alpha^{-1} P^{2}\right) e_{a}\right) \\
+\sigma_{1} \delta_{1}^{2} \widehat{x}_{a}^{T} \widehat{x}_{a}+\sigma_{2} \delta_{2}^{2} u^{T} u+\sigma_{3} \delta_{3}^{2} I-2 e_{a}^{T} P z
\end{gathered}
$$

with

$$
\begin{gathered}
\alpha_{0}=\lambda_{1}(1+\lambda), \quad \propto^{-1}=\left(\lambda_{1}^{-1}+\lambda_{2}^{-1}+\lambda_{3}^{-1}\right), \\
\sigma_{1}=\lambda_{1}\left(1+\lambda^{-1}\right)=\left(\frac{\alpha_{0}}{\lambda}\right), \\
\sigma_{2}=\lambda_{2}=\left(\frac{\alpha \alpha_{0} \lambda_{3}}{\alpha\left(\alpha_{0}+\lambda_{3}(1+\lambda)\right)-\alpha_{0} \lambda_{3}}\right), \\
\sigma_{3}=\lambda_{3} .
\end{gathered}
$$

And taking into account (11c) we obtain

$$
\begin{aligned}
2 e_{a}^{T} P z= & 2 e_{a}^{T} P \sigma_{1} \delta_{1}^{2} \frac{\hat{x}_{a}^{T} \widehat{x}_{a}}{2 e_{a y}^{T} e_{a y}} P^{-1} \bar{C}^{T} e_{a y} \\
& +2 e_{a}^{T} P \sigma_{2} \delta_{2}^{2} \frac{u^{T} u}{2 e_{a y}^{T} e_{a y}} P^{-1} \bar{C}^{T} e_{a y} \\
& +2 e_{a}^{T} P \sigma_{3} \delta_{3}^{2} \frac{1}{2 e_{a y}^{T} e_{a y}} P^{-1} \bar{C}^{T} e_{a y} \\
= & \sigma_{1} \delta_{1}^{2} \widehat{x}_{a}^{T} \hat{x}_{a}+\sigma_{2} \delta_{2}^{2} u^{T} u+\sigma_{3} \delta_{3}^{2} I .
\end{aligned}
$$

Then, the relation (A.2) becomes

$$
\dot{V} \leq \sum_{i=1}^{r} \mu_{i}(\widehat{x}) e_{a}^{T}\left(\overline{\mathscr{A}}_{i}^{T} P+P \overline{\mathscr{A}}_{i}+\alpha_{0} \delta_{1}^{2} I+\propto^{-1} P^{2}\right) e_{a} .
$$

The condition of stability $\dot{V}(t)<0$ (for all $i=1, \ldots, r$ ) is satisfied if

$$
\overline{\mathscr{A}}_{i}^{T} P+P \overline{\mathscr{A}}_{i}+\alpha_{0} \delta_{1}^{2} I+\alpha^{-1} P^{2}+\alpha^{-1} P^{2}<0 .
$$

The Schur complement of condition (A.6) with variables given in (9) allows writing the LMI (11a).

\section{References}

[1] G. Jiang, G. Chen, and W. K. Tang, "Stabilizing unstable equilibria of chaotic systems from a state observer approach," IEEE Transactions on Circuits and Systems II, vol. 51, no. 6, pp. 281-288, 2004

[2] L. M. Pecora and T. L. Carroll, "Synchronization in chaotic systems," Physical Review Letters, vol. 64, no. 8, pp. 821-824, 1990.

[3] T. L. Carroll and L. M. Pecora, "Synchronizing chaotic circuits," IEEE Transactions on Circuits and Systems, vol. 38, no. 4, pp. 453-456, 1991.

[4] E. N. Lorenz, "Deterministic nonperiodic flow," Journal of the Atmospheric Sciences, vol. 20, no. 2, pp. 130-141, 1963.

[5] J. Yang, G. Hu, and J. Xiao, "Chaos synchronization in coupled chaotic oscillators with multiple positive lyapunov exponents," Physical Review Letters, vol. 80, no. 3, pp. 496-499, 1998.

[6] S. Boccaletti, C. Grebogi, Y.-C. Lai, H. Mancini, and D. Maza, "The control of chaos: theory and applications," Physics Reports, vol. 329, no. 3, pp. 103-197, 2000.

[7] S. Boccaletti, J. Kurths, G. Osipov, D. L. Valladares, and C. S. Zhou, "The synchronization of chaotic systems," Physics Reports, vol. 366, no. 1-2, pp. 1-101, 2002.

[8] E. M. Shahverdiev, "Synchronization in systems with multiple time delays," Physical Review E, vol. 70, no. 6, Article ID 067202, 4 pages, 2004.

[9] J. Xu, L. Min, and G. Chen, "A chaotic communication scheme based on generalized synchronization and hash functions," Chinese Physics Letters, vol. 21, no. 8, pp. 1445-1448, 2004.

[10] J. W. Haefner, Modeling Biological Systems: Principles and Applications, Springer, New York, NY, USA, 2005.

[11] B. Shen, Z. Wang, and X. Liu, "Bounded $H_{\infty}$ synchronization and state estimation for discrete time-varying stochastic complex networks over a finite horizon," IEEE Transactions on Neural Networks, vol. 22, no. 1, pp. 145-157, 2011.

[12] Y. Liu, Z. Wang, J. Liang, and X. Liu, "Synchronization of coupled neutral-type neural networks with jumping-modedependent discrete and unbounded distributed delays," IEEE Transactions on Cybernetics, vol. 43, no. 1, pp. 102-114, 2013.

[13] B. Shen, Z. Wang, and X. Liu, "Sampled-data synchronization control of dynamical networks with stochastic sampling," IEEE Transactions on Automatic Control, vol. 57, no. 10, pp. 26442650, 2012.

[14] D. Ding, Z. Wang, H. Dong, and H. Shu, "Distributed $H_{\infty}$ state estimation with stochastic parameters and nonlinearities through sensor networks: the finite-horizon case," Automatica, vol. 48, no. 8, pp. 1575-1585, 2012.

[15] B. Shen, Z. Wang, Y. S. Hung, and G. Chesi, "Distributed $H_{\infty}$ filtering for polynomial nonlinear stochastic systems in sensor networks," IEEE Transactions on Industrial Electronics, vol. 58, no. 5, pp. 1971-1979, 2011.

[16] H. Dong, Z. Wang, and H. Gao, "Distributed filtering for a class of time-varying systems over sensor networks with quantization errors and successive packet dropouts," IEEE Transactions on Signal Processing, vol. 60, no. 6, pp. 3164-3173, 2012.

[17] H. Dong, Z. Wang, J. Lam, and H. Gao, "Fuzzy-model-based robust fault detection with stochastic mixed time delays and successive packet dropouts," IEEE Transactions on Systems, Man, and Cybernetics B, vol. 42, no. 2, pp. 365-376, 2012.

[18] B. Wanga, J. Wang, and S. M. Zhong, "Impulsive synchronization control for chaotic systems," Procedia Engineering, vol. 15, pp. 2721-2726, 2011. 
[19] C. Tao, C. Yang, Y. Luo, H. Xiong, and F. Hu, "Speed feedback control of chaotic system," Chaos, Solitons and Fractals, vol. 23, no. 1, pp. 259-263, 2005.

[20] Y. Yu, "Adaptive synchronization of a unified chaotic system," Chaos, Solitons and Fractals, vol. 36, no. 2, pp. 329-333, 2008.

[21] C. Li, X. Liao, and K.-W. Wong, "Lag synchronization of hyperchaos with application to secure communications," Chaos, Solitons and Fractals, vol. 23, no. 1, pp. 183-193, 2005.

[22] M. S. Tavazoei and M. Haeri, "Determination of active sliding mode controller parameters in synchronizing different chaotic systems," Chaos, Solitons and Fractals, vol. 32, no. 2, pp. 583-591, 2007.

[23] J. G. Barajas-Ramírez, G. Chen, and L. S. Shieh, "Fuzzy chaos synchronization via sampled driving signals," International Journal of Bifurcation and Chaos in Applied Sciences and Engineering, vol. 14, no. 8, pp. 2721-2733, 2004.

[24] T. Liao and S. Tsai, "Adaptive synchronization of chaotic systems and its application to secure communications," Chaos, solitons and fractals, vol. 11, no. 9, pp. 1387-1396, 2000.

[25] C.-J. Cheng, "Robust synchronization of uncertain unified chaotic systems subject to noise and its application to secure communication," Applied Mathematics and Computation, vol. 219, no. 5, pp. 2698-2712, 2012.

[26] T. Yang, "Secure communication via chaotic parameter modulation," IEEE Transactions on Circuits and Systems I, vol. 43, no. 9, pp. 817-819, 1996.

[27] H. Dedieu, M. P. Kennedy, and M. Hasler, "Chaos shift keying: modulation and demodulation of a chaotic carrier using selfsynchronizing Chua's circuits," IEEE Transactions on Circuits and Systems II, vol. 40, no. 10, pp. 634-642, 1993.

[28] K. M. Cuomo, A. V. Oppenheim, and S. H. Strogatz, "Synchronization of Lorenz-based chaotic circuits with applications to communications," IEEE Transactions on Circuits and Systems II, vol. 40, no. 10, pp. 626-633, 1993.

[29] T. Takagi and M. Sugeno, "Fuzzy identification of systems and its applications to modeling and control," IEEE Transactions on Systems, Man and Cybernetics, vol. 15, no. 1, pp. 116-132, 1985.

[30] K. Lian, T. Chiang, C. Chiu, and P. Liu, "Synthesis of fuzzy model-based designs to synchronization and secure communications for chaotic systems," IEEE Transactions on Systems, Man, and Cybernetics B, vol. 31, no. 1, pp. 66-83, 2001.

[31] J.-H. Kim, C.-W. Park, E. Kim, and M. Park, "Adaptive synchronization of T-S fuzzy chaotic systems with unknown parameters," Chaos, Solitons and Fractals, vol. 24, no. 5, pp.13531361, 2005.

[32] C.-H. Hyun, C.-W. Park, J.-H. Kim, and M. Park, "Synchronization and secure communication of chaotic systems via robust adaptive high-gain fuzzy observer," Chaos, Solitons and Fractals, vol. 40, no. 5, pp. 2200-2209, 2009.

[33] S. Boyd, L. El Ghaoui, E. Feron, and V. Balakrishnan, Linear Matrix Inequalities in System and Control Theory, SIAM Studies in Applied Mathematics, Society for Industrial and Applied Mathematics, Philadelphia, Pa, USA, 1994.

[34] K. Tanaka and H. O. Wang, Fuzzy Control System Design and Analysis. A Linear Matrix Inequality Approach, John Wiley \& Sons, New York, NY, USA, 2001.

[35] M. Chadli and H. R. Karimi, "Robust observer design for unknown inputs Takagi-Sugeno models," IEEE Transactions on Fuzzy Systems, vol. 21, no. 1, pp. 158-164, 2013.

[36] H. Dimassi, A. Loria, and S. Belghith, "A new secured transmission scheme based on chaotic synchronization via smooth adaptive unknown-input observers," Communications in Nonlinear Science and Numerical Simulation, vol. 17, no. 9, pp. 37273739, 2012.

[37] M. Chen and W. Min, "Unknown input observer based chaotic secure communication," Physics Letters A, vol. 372, no. 10, pp. 1595-1600, 2008.

[38] M. Chadli and I. Zlinka, "Unknown input observer design for fuzzy systems with application to chaotic system reconstruction," Computers and Mathematics with Applications, vol. 66, no. 2, pp. 147-154, 2013.

[39] M. Chadli, A. Akhenak, J. Ragot, and D. Maquin, "State and unknown input estimation for discrete time multiple model," Journal of the Franklin Institute, vol. 346, no. 6, pp. 593-610, 2009.

[40] T. Youssef, M. Chadli, H. R. Karimi, and M. Zelmat, "Design of unknown inputs proportional integral observers for TS fuzzy models," Neurocomputing Journal, vol. 210, no. 163, p. 174, 2013.

[41] X. Meng, Y. Yu, G. Wen, and R. Chen, "Chaos synchronization of unified chaotic system using fuzzy logic controller," in Proceedings of the IEEE International Conference on Fuzzy Systems (FUZZ '08), pp. 544-547, June 2008.

[42] K. Tanaka, T. Ikeda, and H. O. Wang, "A unified approach to controlling chaos via an LMI-based fuzzy control system design," IEEE Transactions on Circuits and Systems I, vol. 45, no. 10, pp. 1021-1040, 1998. 


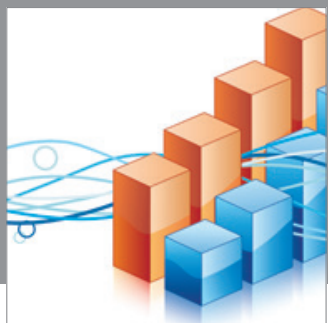

Advances in

Operations Research

mansans

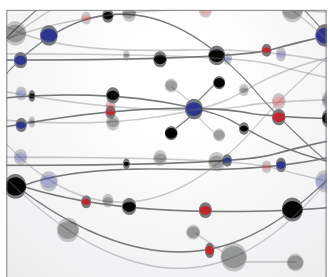

The Scientific World Journal
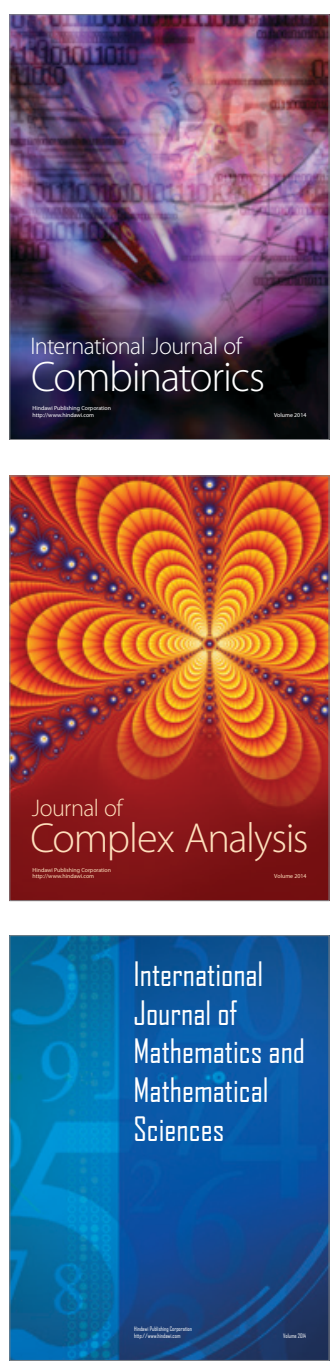
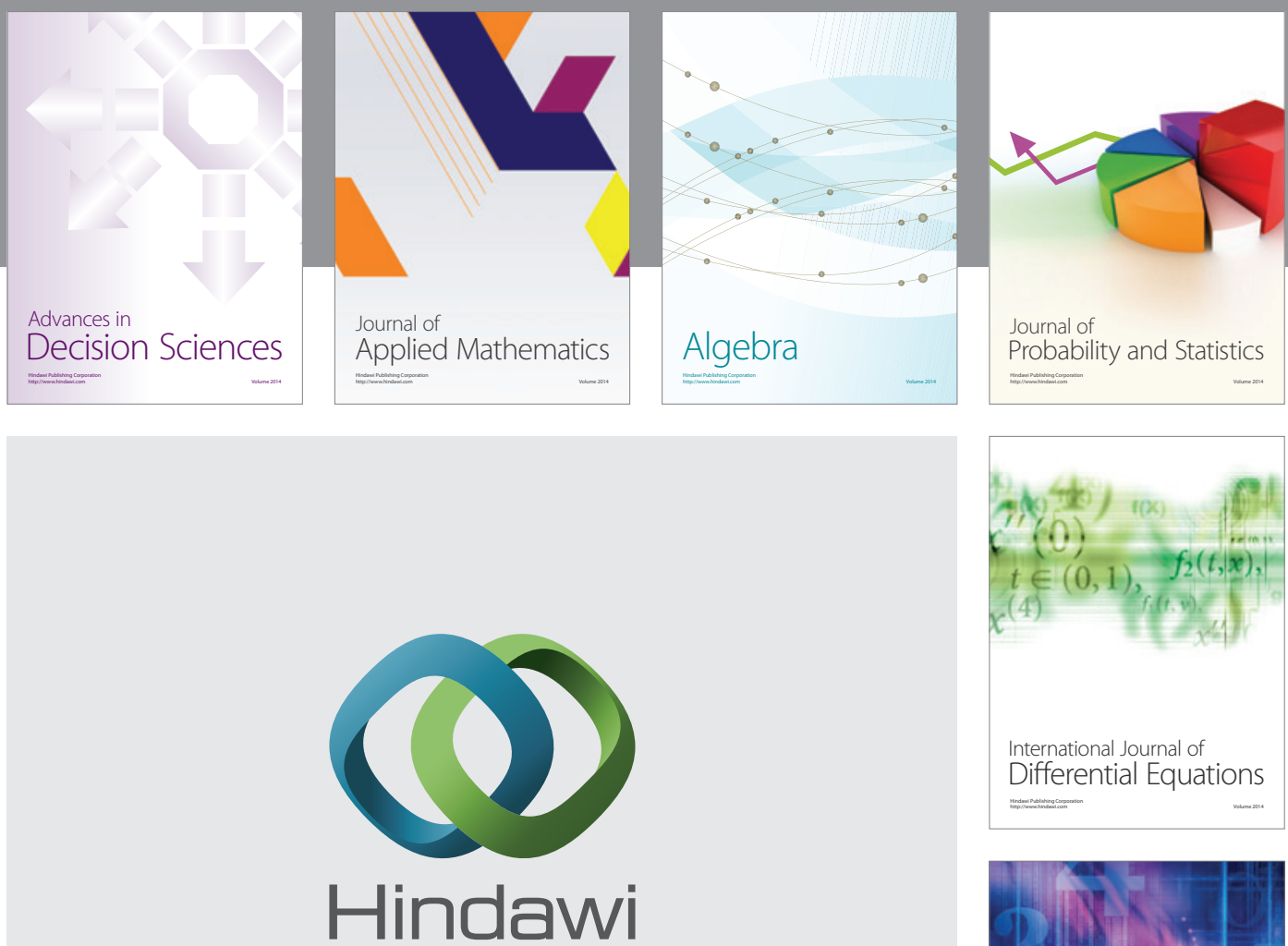

Submit your manuscripts at http://www.hindawi.com
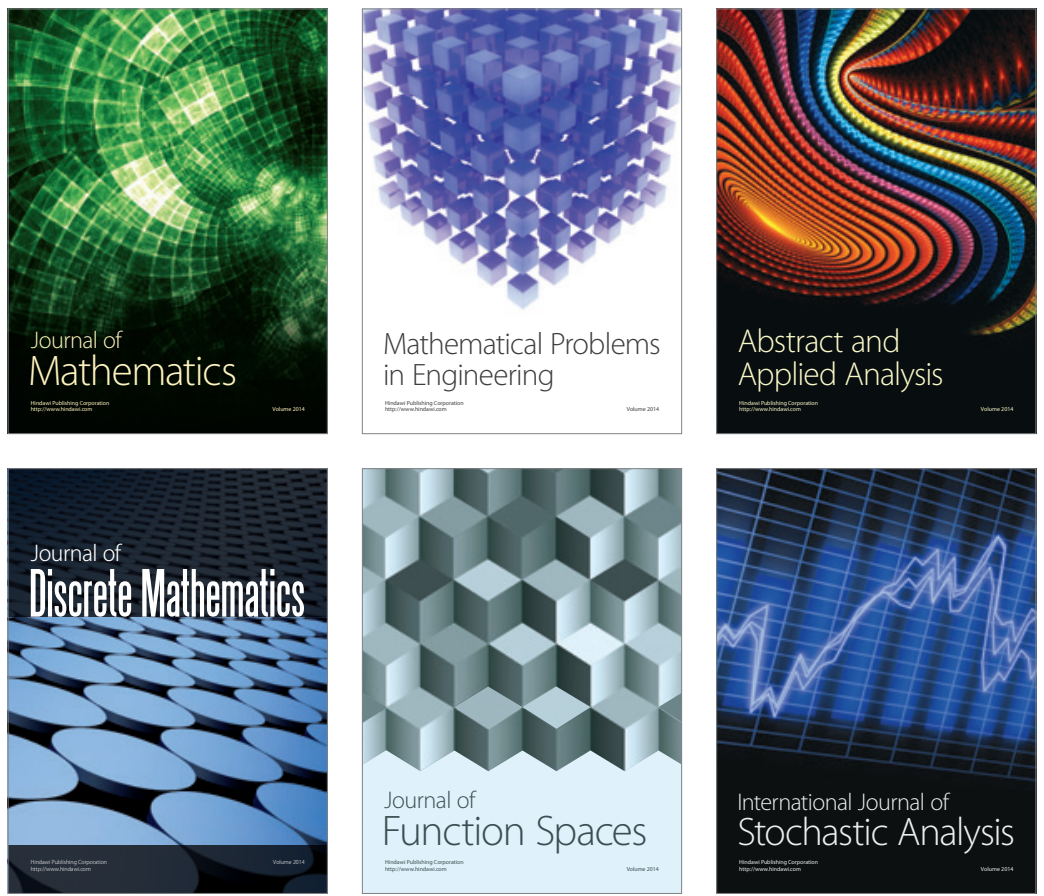

Journal of

Function Spaces

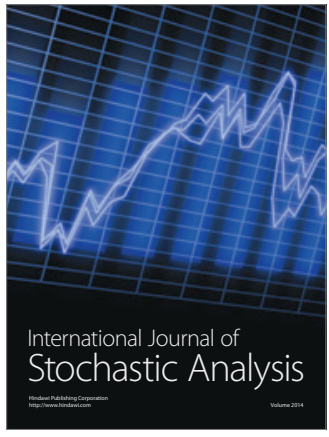

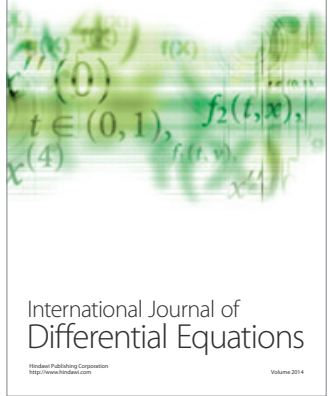
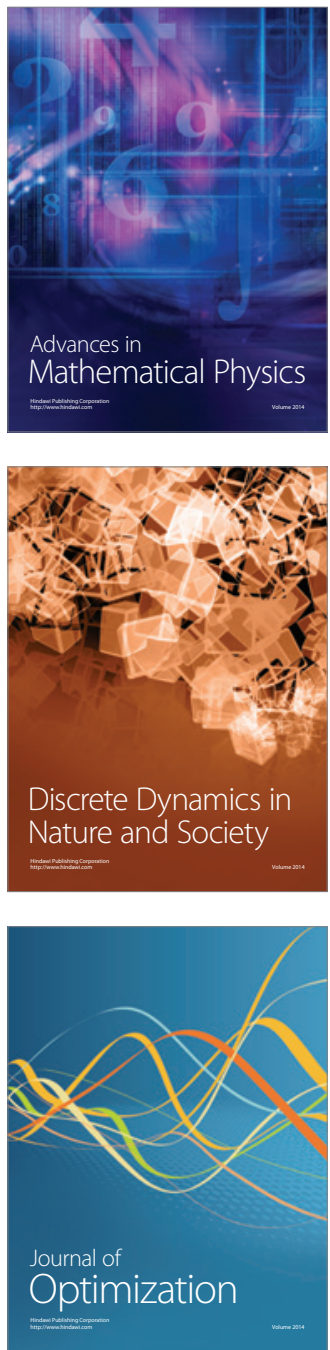